Ręba Patrycja. Stroke - diagnosis and treatment methods. Journal of Education, Health and Sport. 2020;10(5):169-174. eISSN 23918306. DOI http://dx.doi.org/10.12775/JEHS.2020.10.05.017

https://apcz.umk.pl/czasopisma/index.php/JEHS/article/view/JEHS.2020.10.05.017

https://zenodo.org/record/3855670

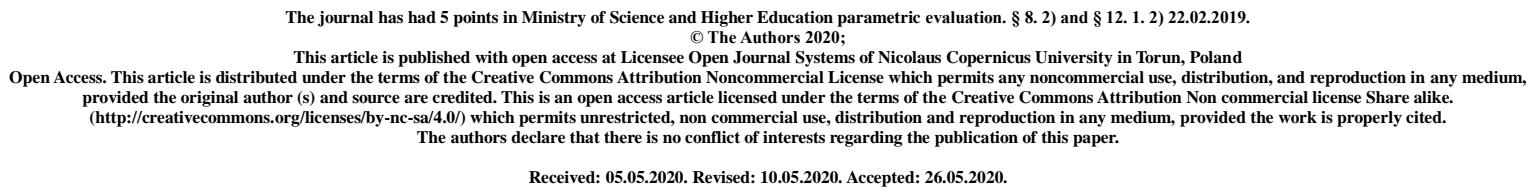

\title{
Stroke - diagnosis and treatment methods
}

\section{Patrycja Ręba}

Faculty of Medicine and Health Sciences, Jan Kochanowski University in Kielce, Poland

\begin{abstract}
Introduction

Stroke is a very serious health problem. Its typical consequences include chronic physical and mental disability, more than half of all patients who survived stroke are dependent, depending on the help of other people even in everyday activities. Stroke is the death of parts of this organ as a result of stopping the blood supply to the brain tissue. There are two main types of stroke: ischemic and hemorrhagic.
\end{abstract}

Aim

The aim of the study is to analyze treatment methods and diagnose stroke.

Material and method

Review of available literature on the subject.

Results

Ischemic stroke (about $4 / 5$ of stroke cases) occurs when an artery supplying some part of the brain with blood becomes obstructed, i.e. blood does not flow through it or it flows from insufficient amount so that brain cells receive as much as they need. Atherosclerosis is the 
most common cause of narrowing or blockage of the artery. Hemorrhagic stroke (about 1/5 of stroke cases) occurs as a result of a cerebral artery wall rupture and blood spilling out of the vessel, as a result of which the blood does not reach the brain tissue in the area supplied by the ruptured artery. The blood escaping from the damaged vessel destroys the surrounding nervous tissue and causes an increase in pressure inside the skull, which disrupts the functioning of the entire brain. Hemorrhagic strokes are divided into intracerebral hemorrhages and subarachnoid hemorrhage.

According to the World Health Organization, around 15 million cases of stroke are diagnosed worldwide each year. Of this group, approximately 5 million die and another 5 million suffer from permanent disability.

\section{Conclusions}

Anyone suspected of having a stroke should be taken to hospital immediately and under specialist medical care. Stroke is a medical emergency and the chances of recovery are greater the sooner treatment is started. Stroke, as the most important cause of chronic disability, is a disease that heavily burden the budget of healthcare systems.

\section{Key words: Stroke; diagnosis; treatment methods}

Stroke is a very serious health problem $[1,2,3]$. Its typical consequences include chronic physical and mental disability, more than half of all patients who survived stroke are dependent, depending on the help of other people even in the field of daily activities $[4,10]$. According to the data of the National Stroke Association, $10 \%$ of survivors with stroke have almost total improvement, $25 \%$ have minimal disability, $40 \%$ of cases have moderate or severe disability and require special care, $10 \%$ people require placement in a Social Welfare Home or other 24-hour long-term care facility, and finally about $15 \%$ of patients die shortly after a stroke $[4,10]$.

Stroke occurs when a blood vessel supplying the brain with oxygen and nutrients breaks (hemorrhagic stroke) or is blocked by a blood clot or other object (acute ischemic stroke; it accounts for about $85 \%$ of all cases [1,2,3]. stroke, usually within a few minutes, nerve cells die in the immediate vicinity of the damaged vessel Stroke is a very serious disease with an acute onset, caused by sudden impairment of blood supply to the brain, which can lead to nerve damage $[1,11]$.

Stroke is the death of parts of this organ as a result of stopping the blood supply to the brain tissue. There are two main types of stroke: ischemic and hemorrhagic.

Ischemic stroke (about 4/5 cases of stroke) occurs when an artery supplying some part of the brain with blood becomes obstructed, i.e. blood does not flow through it or it flows from insufficient amount so that brain cells receive it as much as they need. Atherosclerosis is the most common cause of narrowing or blockage of the artery. So-called atherosclerotic plaques the artery lumen gradually builds up and narrows more and more, until eventually it is completely blocked, usually as a result of a blood clot at the site of narrowing (then the blood flow stops suddenly). Another common cause of clogging of the artery is its embolism, i.e. blockage by a thrombus formed in the heart or, sporadically, in the veins of the limbs - in people who have a patent connection between the right and left atrium of the heart (so-called 
persistent oval hole). Embolism can also occur when fragments of a ruptured atherosclerotic plaque, e.g. in the carotid arteries, break off, reach the cerebral artery with a current of blood, and clog it. When the artery was spontaneous, before the ischemic brain cells were irreversibly destroyed, we deal with a "would-be" stroke - an attack of transient cerebral ischemia $[3,11$,

Hemorrhagic stroke (about 1/5 of stroke cases) occurs as a result of a cerebral artery wall rupture and blood spilling out of the vessel, as a result of which the blood does not reach the brain tissue in the area supplied by the ruptured artery. The blood escaping from the damaged vessel destroys the surrounding nervous tissue and causes an increase in pressure inside the skull, which disrupts the functioning of the entire brain. Hemorrhagic strokes are divided into intracerebral hemorrhages (the damaged vessel is located inside the brain) and subarachnoid(the damaged vessel is on the surface of the brain and blood accumulates between the brain and the protective membrane surrounding it, called the spider tire). The main cause of intracerebral hemorrhage is perennial hypertension, causing the formation of so-called micro aneurysms (dilatations of small arteries) whose walls are prone to rupture. Subarachnoid hemorrhage it is usually caused by a rupture of a larger aneurysm or hemangioma, resulting from a congenital defect in the vessel wall. Hemorrhagic strokes are usually more severe than ischemic strokes, more often they end in death or severe disability, and the treatment options are more limited [5, 12].

A typical element of the post-stroke care system is a high level of unmet needs and expectations. In the context of acute stroke, the available treatment options and long-term prognosis are primarily a function of time, preferably measured in minutes, in the worst case in hours. The risk of stroke depends on many factors [1]. The most important are stroke, transient ischemic incident (TIA), high blood pressure, physical inactivity, old age, diabetes, heart disease and smoking $[1,3]$. The doctor recognizes stroke based on the symptoms and the picture computed tomography or magnetic resonance imaging of the head. Immediate computed tomography imaging makes it possible to distinguish between ischemic and hemorrhagic stroke and determine whether the patient has edema brain or other lesions requiring special treatment. In the early stages of stroke, you don't usually see how much of the brain has been damaged. It will show computed tomography after a few days and weeks. Even more accurately, the area of stroke can be assessed by magnetic resonance imaging. Other tests, such as ultrasound carotid arteries, echocardiography and arteriography of cerebral arteries and blood tests. They are used to determine the cause of stroke [1, 9, 12].

Initial treatment is to protect basic vital functions. Your doctor may decide, for example, to connect a drip (for medication or to supplement fluids and nutrients), to give oxygen for breathing through a mask (to increase the oxygen supply to the brain), or to connect a respirator, i.e. a breathing aid device (if you have difficulty with breathing) $[10,11,12]$.

If less than 3 hours have passed since the onset of the stroke (first symptoms appeared) (in special cases up to 6 hours), it is possible to use tissue plasminogen activator (tPA, alteplase) a medicine that dissolves an artery clot. However, this treatment impairs general blood clotting and is associated with a risk of bleeding. They are used only in patients with ischemic stroke. Alteplase cannot therefore be used in the case of hemorrhagic stroke or in patients who have recently undergone surgery (haemorrhage may occur).wounds) $[9,12]$. 
However, if the artery remains clogged for more than a few hours, hypoxic brain cells irreversibly change and die. Treatment with tissue plasminogen activator is no longer useful. It should be remembered that the onset of stroke is defined as the last time point when the patient was definitely healthy (i.e. had no symptoms of stroke). Because in about a third of cases, stroke occurs during sleep, when the patient notices symptoms (after waking up), it is often too late to start treatment with tissue plasminogen activator. If the patient has not received alteplase, the doctor usually prescribes aspirin (acetylsalicylic acid) and sometimes heparin $[3,6,11]$.

For hemorrhagic stroke, the above-mentioned drugs are not used. If a stroke caused a dangerous increase in intra-cranial pressure, neurosurgical surgery may be necessary. When the cause of intracranial bleeding is a hemangioma of the brain, sometimes an intravascular procedure is performed $[8,11,12]$.

As soon as the patient's condition stabilizes, proper care of the patient is necessary depending on the existing neurological disorders, e.g. adequate nutrition, prevention bedsoresand intensive rehabilitation. These treatments are fundamental to reducing disability after a stroke. In the initial period of stroke in a patient who is unconscious or has a paralyzed limb, rehabilitation consists of proper positioning and changes in body position to prevent pressure sores and contractures. As the patient's condition improves, the rehabilitation program is gradually expanded to include physical exercises and physiotherapeutic procedures that improve mobility and general well-being of the patient. The sick man prepares to sit in bed and walk. In patients with paresis of limbs, learning to walk with a walker or walking stick and exercises to improve the efficiency of the fingers may be needed $[1,7,11]$.

In some patients, the effect of stroke may be aphasia, or difficulties in speaking or understanding speech caused by damage to the speech center in the brain. A patient suffering from aphasia may have difficulty naming objects, cannot remember the names of friends or cannot build sentences correctly. The speech therapist provides assistance to patients with aphasia [11, 12].

Rehabilitation treatment initiated in the acute period of stroke may later be continued in a specialized neurological rehabilitation department. The purpose of staying in such a ward is, among others occupational therapy and learning to function in everyday life and adaptation to work despite possible restrictions resulting from neurological disorders remaining after stroke. Psychotherapy makes it easier to overcome emotional difficulties. Associations of the sick also provide help [12].

Anyone suspected of having a stroke should be taken to hospital immediately and under specialist medical care. Stroke is a medical emergency and the chances of recovery are greater the sooner treatment is started [5,9]. The success of caring for an acute stroke victim depends on a chain of six elements:

- Recognize: quick recognition of heraldic symptoms.

- $\quad$ React: immediate call for an ambulance.

- $\quad$ React for the second time (responder): prioritize treatment, transport to the appropriate facility, advance notification

- $\quad$ Recognize for the second time (reveal): fast and accurate diagnosis with the help of neuroimaging techniques.

- $\quad$ Prescription $(\mathrm{Rx})$ : treatment in a hospital. 
- $\quad$ Rehabilitation (rehabilitation) [9].

A stroke is typical for a stroke, symptoms appear in seconds or minutes, and their exact picture depends on the location and size of the damaged area - the larger, the greater the level of disability expected [1]. According to the recommendations of the American Stroke Association, the most significant harbingers include:

- Sudden sensory disturbance or weakness of the muscles in the face, arms, legs, especially on one side of the body.

- $\quad$ Sudden confusion, difficulty speaking or understanding.

- $\quad$ Sudden vision problems in one or both eyes.

- $\quad$ Sudden gait disturbance, dizziness, imbalance or coordination of movements.

- $\quad$ Sudden, severe headache with no known cause [5, 6].

According to the World Health Organization, around 15 million cases of stroke are diagnosed worldwide each year [2]. Of this group, around 5 million die and another 5 million suffer from permanent disability, which makes stroke the second most common cause of death and one of the most important causes of disability $[6,7]$. Stroke can develop regardless of the age or sex of the patient, but most cases affect people over 65 years of age. In women and men over 55 years old, the risk of stroke doubles with each subsequent decade of life [7]. Symptoms may worsen and then, over several hours or more, disappear partly or completely, and then intensify again. If symptoms resolve completely within 24 hours, an attack of transient cerebral ischemia is recognized.

Based on data collected in seven countries (United States, France, Germany, Italy, Spain, United Kingdom and Japan), the average incidence of stroke was estimated at 214 cases / 100,000 inhabitants / year, with an observed increase of $1.9 \%$ / year related to aging societies. In the United States a new case of stroke is recorded every 40 seconds, resulting in around 2,200 new cases per day [8].

Stroke, as the most important cause of chronic disability, is a disease that heavily burden the budget of healthcare systems. Only in the United States was the annual cost of stroke estimated at USD 65.5 billion in 20086, while in the European Union it was EUR 27 billion [6].

Over the past 20 years, significant progress has been made in the quality of care for stroke patients. The most important achievements include:

1. Establishing a system of hospital stroke wards, in routine conditions it is there where the care of patients with stroke is carried out.

2. Availability of drugs called 'thrombolytics' that treat the acute phase of ischemic stroke by dissolving blood clots blocking the lumen of a blood vessel.

3. Introduction of stroke prevention methods and programs [9].

Thanks to these improvements and advances in diagnostic imaging techniques, a much larger percentage of patients are likely to receive treatment, and the distant results of this treatment have improved significantly. Each stroke, even with minor symptoms, is a life-threatening condition and requires the fastest possible treatment in a hospital, preferably on so-called a stroke ward specializing in caring for stroke patients. This also applies to a seizure of transient cerebral ischemia because it very often precedes stroke for several hours or days $[6,9,11]$.

Brain cells are very sensitive to hypoxia and begin to die as soon as 4 minutes after the onset of stroke. Many patients qualify for treatment that can reverse or significantly reduce the 
effects of stroke, provided that this treatment is given no later than 3 hours after the onset of stroke. So every second counts, and the most important thing you can and should do if you suspect you or someone else having a stroke is to call an ambulance as soon as possible. Getting to the hospital as soon as possible can save your life or save you from severe disability $[4,6,12]$.

\section{Literature:}

1. National Institute of Neurological Disorders and Stroke (NINDS). Stroke: Hope through research 2011 [www.ninds.nih.gov/disorders/stroke/detail_stroke.htm. 08/04/20].

2. Mackay J, Mensah G (eds.). The atlas of heart disease and stroke. Global burden of stroke. World Health

Organization

(WHO) 2004 [http://www.who.int/cardiovascular_diseases/en/cvd_atlas_15_burden_stroke.pdf. 07/04/20]. 3. Roger VL, Go AS, Lloyd-Jones DM, et al. Heart disease and stroke statistics - 2011 update: a report from the American Heart Association. Circulation 2011; 123 (4): e18 - e209.

4. National Stroke Association (NSA). Rehabilitation therapy after stroke [http://www.stroke.org 04/03/20].

5. American Stroke Association. Warning signs. Last updated 2007 [http://www.strokeassociation.org/STROKEORG/WarningSigns/WarningSigns_UCM_308528_SubHomePage.jsp. 04/13/20].

6. Di Carlo A. Human and economic burden of stroke. Age Aging 2009; 38 (1): 4-5.

7. Feigin VL, Lawes CM, Bennett DA, Anderson CS. Stroke epidemiology: a review of population-based studies of incidence, prevalence, and case-fatality in the late 20th century. Lancet Neurol 2003; 2 (1): 43-53.

8. Searles JW, Avodele L, Kuhlmann GL. Acute ischemic stroke. Decision Resources.Waltham, Massachusetts, USA: 2009.

9. National Institute of Neurological Disorders and Stroke (NINDS). Stroke proceedings: Pepe overview.

2011

[www.ninds.nih.gov/news_and_events/proceedings/stroke_proceedings/pepeover.htm. 10/4/20].

10. Hirtz D., Thurman DJ, Gwinn-Hardy K. et al. How common are the "common" neurologic disorders. Neurology, 2007, 68, 326-33.

11. Mazur R., Książkiewicz B .: Clinical symptomatology of acute vascular strokes the brain. Cerebrovascular accident. PZWL, Warsaw 1998.

12. Poeck K .: Neurology. Springer Verlag, Berlin 1994. 